Lembi, R.C.; Gonçalves, L.R.; Seixas, C.S. Panorama dos projetos de turismo em Unidades de Conservação na Mata Atlântica. Revista Brasileira de Ecoturismo, São Paulo, v 15, n.1, fev-abr 2022, pp. 45-63.

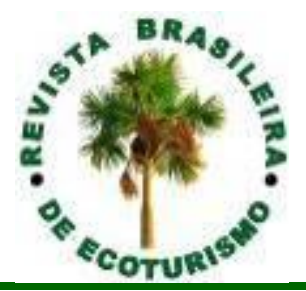

\title{
Panorama dos projetos de turismo em Unidades de Conservação na Mata Atlântica
}

\section{Panorama of tourism projects in Protected Areas within the Atlantic Forest}

\author{
Rafael Cavalcanti Lembi, Leandra Regina Gonçalves, Cristiana Simão Seixas
}

\begin{abstract}
RESUMO: O turismo na natureza é uma atividade importante para a economia e qualidade de vida das pessoas, e é frequentemente praticado em Unidades de Conservação (UCs). No Brasil, as UCs localizadas no bioma da Mata Atlântica concentram a maior porção do turismo em UCs. Neste contexto, intervenções como a implementação de projetos podem ser uma ferramenta para fomentar o turismo nestas áreas, podendo atuar na conciliação entre conservação e desenvolvimento. Visando contribuir para o planejamento e gestão do turismo, o presente trabalho de revisão bibliográfica objetiva descrever um panorama dos projetos de turismo em UCs na Mata Atlântica, e avaliar como o turismo tem sido fomentado neste recorte. No total, 26 projetos foram identificados e analisados. Os resultados obtidos revelam uma concentração geográfica de projetos de turismo em UCs da região sudeste do Brasil, em especial no estado de São Paulo. Os Parques foram a categoria de UC mais visadas para intervenções. Os projetos possuíam, em geral, apenas uma fonte de financiamento, com destaque para órgãos governamentais. No que se refere ao planejamento e execução, organizações da sociedade civil (ONGs e OSCIPs) e associações comunitárias se destacaram. Os projetos possuíam diferentes eixos de atuação e objetivos que foram agrupados em três categorias: gestão, capacitação e infraestrutura. Ao descrever um panorama de projetos de turismo em UCs na Mata Atlântica, o presente estudo contribui para elucidar como a atividade turística tem sido planejada neste recorte geográfico, podendo ser útil para o embasamento de novas intervenções guiadas pelos princípios do ecoturismo.
\end{abstract}

PALAVRAS-CHAVE: Áreas Protegidas; Gestão; Uso Público; Ecoturismo; Turismo Na Natureza.

ABSTRACT: Nature tourism is an important activity for the economy and to people's quality of life and is frequently practiced in Protected Areas (PAs). In Brazil, PAs located in the Atlantic Forest biome concentrate the largest portion of tourism in PAs. In this context, interventions such as the implementation of projects can be a tool to promote tourism in these areas and can act in reconciling conservation and development. Aiming to contribute to the planning and management of tourism, the present literature review focuses on describing an overview of tourism projects in PAs within the Atlantic Forest, and to assess how tourism has been promoted in this context. In total, 26 projects were identified and analysed. Obtained results reveal a geographical concentration of tourism projects in PAs in the South-eastern region of Brazil, especially in the state of São Paulo. Parks were the most targeted category for interventions. Projects generally had only one source of funding, with an emphasis from government agencies. Regarding planning and execution, civil society organizations (NGOs and "OSCIPs") and community associations stood out. The projects had different lines of action and objectives that were grouped into three categories: management, training and infrastructure. By describing an overview of tourism projects in PAs in the Atlantic Forest, the present study contributes to elucidate how tourism activities have been planned in this geographic section and can be useful to support new interventions guided by the principles of ecotourism.

KEYWORDS: Protected Areas; Management; Public Use; Ecotourism; Nature Tourism. 


\section{Introdução}

O turismo é uma das mais importantes atividades econômicas mundiais. É um dos poucos setores que teve plena expansão entre 2010 e 2019, apresentando crescimento econômico ininterrupto e movimentando globalmente cerca de US\$ 5 bilhões por dia (UNWTO, 2019). Em ambientes naturais, o turismo segue tendência semelhante de crescimento. No Brasil, as atividades de contato com a natureza têm aumentado tanto em relação aos seus tipos, como em relação ao número de pessoas (BRASIL, 2009). Em 2017, a visitação em Unidades de Conservação (UCs) de administração federal, por exemplo, atingiu o patamar de 12 milhões de pessoas (ICMBIO, 2019), gerando um valor agregado ao PIB de $R \$ 3,1$ bilhões e cerca de 80 mil empregos (SOUZA; SIMÕES, 2018). Há ainda que considerar que nem todas as UCs possuem registro de visitantes, o que pode significar números ainda maiores. A exceção tem sido o ano de 2020, no qual a pandemia de covid-19 impôs medidas de isolamento social que levaram à suspensão da visitação pública em UCs (ICMBIO, 2020) e o ano de 2021 em que os efeitos da pandemia se estenderam.

Por meio da gestão apropriada, as atividades de recreação e lazer podem se tornar uma potencial fonte de ganhos financeiros para as áreas protegidas, podendo, em certos países, até mesmo promover um autofinanciamento para aquelas com poucos recursos financeiros (DHARMARTNE et al., 2000). Em um contexto local, a atividade turística também se revela como uma importante estratégia para se garantir meios de subsistência e renda para comunidades tradicionais (e.g., PRADO et al., 2015).

Para além dos benefícios econômicos, experiências físicas e psicológicas na natureza influenciam a saúde física e mental, e práticas como a visitação em UCs podem ser consideradas contribuições imateriais da natureza para o bem-estar de seus visitantes (DÍAZ et al., 2018; BRATMAN et al., 2019). UCs também se revelam como locais ideais para a implementação de programas educativos, por proverem uma fonte inesgotável de oportunidades que facilitam o religar do homem ao ambiente (WWF BRASIL, 2003). Neste sentido, podem atuar como verdadeiras fábricas de remodelação do comportamento dos turistas, sensibilizando-os em suas atitudes de forma a se tornarem viajantes e consumidores mais conscientes socioambientalmente (LUO; DENG, 2007).

No Brasil, a Mata Atlântica é o bioma mais visitado. No ano de 2018 foram registrados 7,6 milhões de visitantes formais nas UCs federais da Mata Atlântica, totalizando cerca de $64 \%$ do total da visitação para o país (ICMBIO, 2019). O bioma é classificado como um "hotspot" para a conservação, devido à riqueza de sua biodiversidade, elevado endemismo de espécies e alto grau de ameaça em que se encontra (REZENDE et al., 2018). Deste modo, fica evidente a importância do turismo em suas 1437 UCs (BRASIL, 2020).

Projeções de Souza e Thapa (2018) indicam que as UCs potencialmente poderiam receber um maior número de turistas. O aumento da visitação, entretanto, pode ter impacto negativos nas UCs e qualidade de vida das comunidades locais, geralmente de populações tradicionais, que vivem dentro ou ao redor destas áreas protegidas (SPINOLA, 2006). Evidencia-se, portanto, a necessidade do planejamento e gestão da recreação e lazer.

A atividade turística dentro de UCs deve ser pautada nos princípios do ecoturismo: conservação da natureza, desenvolvimento socioeconômico, educação ambiental e o bem-estar de visitantes e populações locais (BRASIL, 2010). Projetos e 
intervenções podem fomentar o turismo através de práticas baseadas nestes princípios. Deste modo, uma revisão sistemática sobre como projetos de turismo tem sido implementados em UCs na Mata Atlântica pode elucidar como a atividade turística tem sido planejada neste recorte. Tais resultados podem informar como a gestão do turismo tem sido fomentada, sendo potencialmente útil para informar a formulação de futuros projetos e políticas públicas que visem estimular o ecoturismo. Portanto, o presente trabalho objetiva:

(i) descrever um panorama dos projetos de turismo em Unidades de Conservação na Mata Atlântica; e,

(ii) avaliar como o turismo tem sido fomentado e implementado nestas Unidades de Conservação.

\section{Metodologia}

Neste trabalho, adota-se a delimitação geográfica do bioma Mata Atlântica conforme Rezende et al. (2018), baseada em dados do governo federal. Aqui, projetos de turismo são definidos como planos ou intervenções em UCs com um objetivo relacionado ao turismo.

Um levantamento bibliográfico foi realizado através do mecanismo de busca e combinação de palavras-chave (DIAS, 2016), nas ferramentas de busca Google Acadêmico (literatura científica) e Google (literatura cinza) entre os meses de junho e julho de 2018. Ao todo, foram realizadas 6 combinações de palavras-chave na língua portuguesa (Quadro 1), e para cada uma das combinações possíveis foram avaliados os 50 primeiros retornos.

Quadro 1: Combinações de palavras-chaves efetuadas para busca no Google Acadêmico e Google.

Box 1: Key words combinations utilized for the search in Google Scholar and Google.
"projetos de turismo" Unidades de Conservação mata atlântica brasil
"projetos de turismo" áreas protegidas mata atlântica brasil
"projetos de turismo" Unidades de Conservação floresta atlântica brasil
"projetos de turismo" áreas protegidas floresta atlântica brasil
visitação projetos Unidades de Conservação mata atlântica brasil
turismo projetos Unidades de Conservação mata atlântica brasil

Os 600 retornos resultantes das 6 combinações nas duas bases de dados foram analisados, e alguns foram selecionados como condizentes com os objetivos da pesquisa. Os critérios de seleção foram: (i) ser um projeto que buscava desenvolver algum aspecto referente ao turismo, podendo o desenvolvimento do turismo ser um objetivo principal ou secundário; (ii) ser um projeto em Unidades de Conservação localizadas no recorte geográfico do domínio do bioma da Mata Atlântica.

A partir das informações disponíveis nas bases de dados, os projetos selecionados foram caracterizados de acordo com: estado(s) de abrangência, tipo de Unidade(s) de Conservação, tipo de financiamento, principais organizações envolvidas no planejamento e execução, e eixo(s) de atuação do projeto. 


\section{Resultados e discussão}

A partir do levantamento bibliográfico efetuado, foi possível identificar um total de 26 projetos de turismo em UCs localizadas no bioma da Mata Atlântica. Os projetos foram numerados e compilados na Tabela 1, e caracterizados em detalhe no Apêndice 1.

Tabela 1: Projetos selecionados de turismo em Unidades de Conservação na Mata Atlântica.

Table 1: Selected tourism projects in protected areas in the Atlantic Forest.

\begin{tabular}{|c|c|c|}
\hline & Projeto & Referência \\
\hline 1 & $\begin{array}{l}\text { Projeto de Desenvolvimento do Ecoturismo na região } \\
\text { da Mata Atlântica no Estado de São Paulo }\end{array}$ & $\begin{array}{l}\text { KOGA et al. (2013), SÃO PAULO } \\
(2013)\end{array}$ \\
\hline 2 & Programa de Ecoturismo para a Vila das Peças & WANDEMBRUCK (2005) \\
\hline 3 & Projeto Turismo de Base Comunitária & SALVADOR et al. (2013) \\
\hline 4 & $\begin{array}{l}\text { Projeto de Extensão Povo do Aventureiro: } \\
\text { fortalecimento do turismo de base comunitária }\end{array}$ & $\begin{array}{l}\text { COSTA et al. (2009), FERREIRA } \\
(2014)\end{array}$ \\
\hline 5 & Projeto Turístico Socialmente Responsável & MENDONÇA (2009) \\
\hline 6 & Tramas em Riacho Doce & BARTHOLO et al. (2009a) \\
\hline 7 & $\begin{array}{l}\text { Base Local Turismo: promovendo o turismo de base } \\
\text { comunitária na Costa do Cacau, BA }\end{array}$ & BARTHOLO et al. (2009b) \\
\hline 8 & $\begin{array}{l}\text { Estruturação e fortalecimento do turismo de base } \\
\text { comunitária do povoado de Terra Caída }\end{array}$ & BARTHOLO et al. (2009c) \\
\hline 9 & Ecobase Ilha das Caieiras & BARTHOLO et al. (2009d) \\
\hline 10 & $\begin{array}{l}\text { Ecoturismo de base comunitária da região da Trilha do } \\
\text { Ouro }\end{array}$ & BARTHOLO et al. (2009e) \\
\hline 11 & $\begin{array}{l}\text { Turismo sustentável de base comunitária na Reserva } \\
\text { de Desenvolvimento Sustentável Barra do Una }\end{array}$ & BARTHOLO et al. (2009f) \\
\hline 12 & $\begin{array}{l}\text { Deslocamentos: ecoturismo de base comunitária no } \\
\text { litoral norte do Paraná }\end{array}$ & BARTHOLO et al. (2009g) \\
\hline 13 & $\begin{array}{l}\text { Boas práticas de turismo de base comunitária no } \\
\text { Território da Serra do Brigadeiro }\end{array}$ & $\begin{array}{l}\text { MORAES et al. (2017); BARTHOLO et } \\
\text { al. (2009h) }\end{array}$ \\
\hline 14 & $\begin{array}{l}\text { Plano municipal de conservação e recuperação da } \\
\text { Mata Atlântica do município de ltu }\end{array}$ & PREFEITURA DE ITU (2017) \\
\hline 15 & Cooperaguá e o ecoturismo na APA de Guaraqueçaba & SPVS (2011) \\
\hline 16 & $\begin{array}{l}\text { Turismo sustentável nos corredores ecológicos do } \\
\text { estado do Espírito Santo }\end{array}$ & BRASIL (2007) \\
\hline 17 & $\begin{array}{l}\text { Formação e organização de Condutores Ambientais } \\
\text { Locais pelo IFRS, Campus Porto Alegre }\end{array}$ & SILVA et al. (2015) \\
\hline 18 & $\begin{array}{l}\text { Unidades de Conservação da Costa de Araranguá - } \\
\text { difundindo a natureza local com o Roteiro } \\
\text { Geoecológico }\end{array}$ & $\begin{array}{l}\text { FUNDAÇÃO SOS MATA ATLÂNTICA } \\
(2018 \mathrm{a})\end{array}$ \\
\hline 19 & $\begin{array}{l}\text { Programa de abertura de escalada em rocha na } \\
\text { Floresta Nacional de Ipanema, e monitoramento do } \\
\text { Urubu-Rei nos setores de escalada }\end{array}$ & $\begin{array}{l}\text { FUNDAÇÃO SOS MATA ATLÂNTICA } \\
\text { (2018b) }\end{array}$ \\
\hline 20 & $\begin{array}{l}\text { Trilhas da Gralha Azul - Projeto de Turismo de Base } \\
\text { Comunitária com o protagonismo juvenil na Flona do } \\
\text { Assungui - Campo Largo (PR) }\end{array}$ & $\begin{array}{l}\text { FUNDAÇÃO SOS MATA ATLÂNTICA } \\
\text { (2018c) }\end{array}$ \\
\hline 21 & Caminho da Mata Atlântica & $\begin{array}{l}\text { DINIZ (2018); CAMINHO DA MATA } \\
\text { ATLÂNTICA }([\underset{\sim}{[} / \mathrm{d}])\end{array}$ \\
\hline 22 & $\begin{array}{l}\text { Potencializando o uso público das Unidades de } \\
\text { Conservação }\end{array}$ & $\begin{array}{l}\text { CONSERVAÇÃO INTERNACIONAL } \\
\text { BRASIL ([s/d]a) }\end{array}$ \\
\hline 23 & $\begin{array}{l}\text { Projeto de Fortalecimento do Parque Nacional do Pau- } \\
\text { Brasil e entorno }\end{array}$ & $\begin{array}{l}\text { CONSERVAÇẤO INTERNACIONAL } \\
\text { BRASIL }\left(\left[\mathrm{s} / \tilde{\sim}_{\tilde{\sigma}}\right] \mathrm{b}\right)\end{array}$ \\
\hline 24 & $\begin{array}{l}\text { Programa de Ecoturismo e Conservação Na Trilha do } \\
\text { Mico-Leão-Dourado }\end{array}$ & $\begin{array}{ll}\text { ASSOCIACCÃO } & \text { MICO-LEÃO } \\
\text { DOURADO }([\mathrm{s} / \mathrm{d}]) & \end{array}$ \\
\hline 25 & $\begin{array}{l}\text { Consolidação da Gestão do Uso Público nas Unidades } \\
\text { de Conservação Estaduais do Estado do Rio de } \\
\text { Janeiro }\end{array}$ & $\begin{array}{l}\text { PELLEGRINI, } 2013 ; \quad \text { INSTITUTO } \\
\text { TERRA DE } \\
\text { AMBIENTAL ([s/d]) }\end{array}$ \\
\hline 26 & Projeto Ecossistemas Costeiros & LOPES (2017) \\
\hline
\end{tabular}


A distribuição geográfica dos projetos selecionados revela uma concentração na região sudeste (12 projetos, $46 \%$ ), em especial no estado de São Paulo (6 projetos, 23\%) (Tabela 2). Um possível fator explicativo para esta observação é o fato da região sudeste concentrar a maior parte (59\%) das 1437 UCs localizadas na Mata Atlântica (BRASIL, 2020). Outro fator explicativo é a origem das publicações. No âmbito específico do turismo na natureza, segundo levantamento feito por Costa (2015), 34\% das publicações são originárias da região sudeste. Ainda, Neiman et al. (2010) indicam que o Sudeste é a região do país com maior porcentagem de grupos de pesquisa em ecoturismo (48\%). Deste modo, o presente estudo corrobora tal tendência ao identificar um maior número de publicações científicas e de literatura cinza sobre projetos de turismo em UCs na Mata Atlântica neste recorte geográfico.

Tabela 2: Quantidade de projetos de turismo em Unidades de Conservação por região e estados na Mata Atlântica*.

Table 2: Quantity of tourism projects in protected areas per region and states in the Atlantic Forest

\begin{tabular}{l|cl}
\hline Regiões & Número de projetos & Projetos por estado $^{\star \star}$ \\
\hline Nordeste & 5 & CE (1), SE (1), BA (3) \\
Sudeste & 12 & MG (1), ES (2), RJ (3), SP (6) \\
Sul & 6 & PR (4), SC (1), RS (1) \\
Interestadual & 3 &
\end{tabular}

*O Projeto 21 (“Caminho da Mata Atlântica”) não foi considerado nesta tabela por abranger um número elevado de UCs e ainda estar em fase de implementação

${ }^{\star \star}$ Ceará (CE), Sergipe (SE), Bahia (BA), Minas Gerais (MG), Espírito Santo (ES), Rio de Janeiro (RJ), São Paulo (SP), Paraná (PR), Santa Catarina (SC), Rio Grande do Sul (RS)

Em relação às UCs em que foram desenvolvidos estes projetos, dezessete dos projetos tinham foco apenas em uma UC, e nove envolviam mais de uma UC. Entre estes nove projetos, alguns tinham abrangência de apenas duas UCs, como é o caso do "Ecobase llha das Caieiras" (Projeto 9) em Vitória, Espírito Santo (BARTHOLO et al., 2009d). Já o projeto "Caminho da Mata Atlântica" (Projeto 21) envolve mais de 70 UCs, em uma proposta de construir uma "megatrilha" de $3.000 \mathrm{~km}$ através de uma rede interestadual que abrange Rio de Janeiro, São Paulo, Paraná, Santa Catarina e Rio Grande do Sul (DINIZ, 2018).

No que se refere às categorias de UCs em que se desenvolveram os projetos, os Parques foram majoritariamente beneficiados (Tabela 3), sendo também a categoria mais visitada em nível federal na Mata Atlântica (68\% do total de visitantes) (ICMBio, 2019). Tal dado indica que, embora a visitação pública seja permitida mesmo com restrições - em todas as categorias de UCs, a categoria Parque é a que se destaca em atrair visitantes. Especificamente no que se refere às Estações Ecológicas e Reservas Biológicas, a visitação está necessariamente atrelada a fins educativos e de pesquisa, segundo a Lei Federal 9.985/00 que institui o Sistema Nacional de Unidades de Conservação. Por exemplo, o projeto "Turismo sustentável nos corredores ecológicos do estado do Espírito Santo" (Projeto 16) abrange quatro UCs, incluindo a Reserva Biológica de Comboios em Regência (ES), e atuou realizando cursos de capacitação em condução ecoturística, formandos pessoas aptas a atuarem localmente, e as sensibilizando em relação às questões ambientais (BRASIL, 2007). 
Tabela 3: Categoria e quantidade de Unidades de Conservação (UCs) em que se desenvolveram projeto de turismo.

Table 3: Category and quantity of protected areas in which tourism projects were developed.

\section{Categoria de UC}

Parque

Área de Proteção Ambiental

Floresta Nacional

Reserva Biológica

Reserva de Desenvolvimento Sustentável

Reserva Extrativista

Estação Ecológica

\section{Número de UCs}

14

6

3

2

2

Sobre o financiamento dos projetos, nota-se que 10 dos 26 projetos foram financiados exclusivamente através de verba pública federal (Apêndice 1). A maior parte destes 10 projetos foram descritos por Bartholo et al. (2009), em publicação que compilou projetos financiados por um edital do Ministério do Turismo (Edital 01/2008) (Projetos 6-13). Modelos mistos de financiamento também foram identificados, como a chamada proposta pela organização não-governamental Fundação SOS Mata Atlântica em colaboração com a empresa Repsol Sinopec Brasil no Edital 01/2018 que financiou 3 projetos de turismo em UCs (Projetos 18-20) (FUNDAÇÃO SOS MATA ATLÂNTICA, 2018a, 2018b, 2018c). O único projeto com um modelo de financiamento de base comunitária é o "Projeto Turístico Socialmente Responsável" (Projeto 5) desenvolvido na Reserva Extrativista Prainha do Canto Verde (CE). Neste projeto, uma cooperativa comunitária local foi criada com um fundo social advindo de verbas de operações ligadas ao turismo, disponibilizando renda para investimentos em diversos outros pequenos projetos. A comunidade da Prainha do Canto Verde possui histórico de mobilização social e auto-organização (PRADO, 2019), e os laços comunitários influenciam na geração e qualificação de um produto turístico diferenciado no litoral cearense (MENDONÇA, 2009).

O fato de a maior parte dos projetos possuírem financiamento público integral ou parcial evidencia a importância do setor público para o turismo em UCs. Entretanto, conforme aponta Adams et al. (2008), existe um descompasso entre o valor atribuído às UCs pelas pessoas e os investimentos realizados pelo governo. Além disso, a dependência de fontes governamentais para financiamento tem se demonstrado um modelo cada vez mais economicamente insustentável nas diversas áreas protegidas no mundo (WHITELAW et al., 2014). No Brasil, cortes orçamentários nas pastas ambientais governamentais têm ocorrido, com diminuição de $26 \%$ do orçamento destinado ao apoio e gestão de UCs federais apenas no ano de 2019 (RODRIGUES, 2019).

Em uma realidade na qual a visitação em áreas protegidas na Mata Atlântica tem aumentado a cada ano, exceto durante a pandemia, colaborações entre múltiplos atores na gestão e financiamento podem contribuir para que a prática do turismo conserve a natureza e seja economicamente viável (JAMAL; STRONZA, 2009). Whitelaw et al. (2014) apontam uma gama de mecanismos inovadores de geração de receita financeira que podem conciliar conservação e desenvolvimento do turismo em áreas protegidas, a partir de abordagens como pagamento por serviços ambientais, hipotecas ambientais, disposição em pagar por parte dos visitantes, entre outras possibilidades. No caso dos projetos de turismo em UCs na Mata Atlântica, nenhuma destas abordagens foi identificada no levantamento bibliográfico, e o financiamento dos projetos em geral envolve apenas uma instituição (por exemplo, governo federal), 
ou no máximo dois tipos de instituições diferentes (por exemplo, governo estadual e banco internacional, ou governo e organização não-governamental) (Apêndice 1).

No que se refere às instituições proponentes e/ou executoras dos projetos de turismo, nota-se uma predominância das organizações não-governamentais (ONGs) e organizações da sociedade civil de interesse público (OSCIPs) (Tabela 4). Tais organizações aparecem como importantes articuladoras de atividades turísticas entre diversos atores, como no caso do "Projeto de fortalecimento do Parque Nacional do Pau-Brasil”, em Porto Seguro (BA) (Projeto 23). Neste caso, a ONG Conservação Internacional Brasil (Cl Brasil) atua no desenvolvimento do uso público no Parque e sua área de influência, através de ações como organização e consolidação dos atrativos da UC, estímulo de ações de conservação e marketing, e impulso de ações integradas da cadeia produtiva do turismo. O projeto articula contatos entre parceiros como o ICMBio, Prefeitura de Porto Seguro, Senac Porto Seguro e outras UCs (CONSERVAÇÃO INTERNACIONAL BRASIL, [s/d]b). Neste sentido, por ser uma organização que articula estrategicamente atores de múltiplos setores na busca de soluções para um problema, podemos definir as ONGs e OSCIPs atuantes nos projetos como organizações-ponte (BROWN, 1991; CRONA; PARKER, 2012). Especificamente no campo do turismo sustentável, ONGs já foram documentadas como sendo uma importante organização-ponte para conciliar conservação e desenvolvimento (JAMAL et al., 2007).

Tabela 4: Categoria das instituições e quantidade de projetos de turismo em que estavam envolvidas em Unidades de Conservação na Mata Atlântica.

Table 4: Category of institutions and quantity of tourism projects in which they were involved in protected areas in the Atlantic Forest.

\begin{tabular}{lc}
\hline Categoria da instituição & Quantidade de projetos \\
\hline Organizações não-governamentais e Organizações da sociedade civil & 14 \\
de interesse público & 9 \\
Associações comunitárias & 5 \\
Órgãos governamentais & 4 \\
Universidades/Institutos & 2 \\
Empresas & \\
\hline
\end{tabular}

Embora estudos iniciais sobre organizações-ponte tenham focado em colaborações entre ONGs e outros atores, tais organizações podem se apresentar de diversos modos (CRONA; PARKER, 2012). O projeto "Unidades de Conservação da Costa de Araranguá - difundindo a natureza local com o Roteiro Geoecológico" é um exemplo (Projeto 18). Liderado pela empresa privada Geoambiental Brasil, o projeto busca divulgar a existência e a importância das UCs de Araranguá (SC), através da implementação de um roteiro turístico que oferece informações sobre a geologia da região (FUNDAÇÃO SOS MATA ATLÂNTICA, 2018a). Neste caso, fortalece-se o potencial turístico da região utilizando uma proposta educativa, e trabalho em conjunto entre empresa, órgãos públicos e financiadores da proposta.

Tanto no âmbito do planejamento como também do financiamento, colaborações entre poder público e a iniciativa privada têm se mostrado recorrentes no contexto do turismo em UCs. Parcerias público-privadas têm ocorrido através da concessão de prestação de serviços dentro das UCs, e experiências internacionais têm demonstrado sua eficácia financeira e na gestão (EAGLES, 2009). Entretanto, como ressaltam Rodrigues e Godoy (2013), não devem ser vistas como uma substituição das organizações públicas pelas privadas, e devem ser criteriosamente avaliadas antes de serem incentivadas. Em projetos de turismo com a participação de 
múltiplos atores, os diversos setores envolvidos podem se beneficiar através de uma relação de parceria que objetiva benefícios mútuos (WILSON et al., 2009). Isto é particularmente relevante tomando-se em conta que muitas das UCs brasileiras foram criadas em áreas onde existiam - ou ainda existem - comunidades indígenas e tradicionais, evidenciando a necessidade de colaborações.

Embora tratem do tema do turismo, os projetos selecionados para esta pesquisa foram articulados de modo a se alcançar diferentes objetivos. A partir da categorização dos objetivos e/ou resultados obtidos, observou-se três categorias possíveis de eixos de atuação em que os projetos se encaixavam: gestão, capacitação e infraestrutura (TABELA 5). De modo a exemplificar estas categorias, podemos citar o "Projeto de Desenvolvimento do Ecoturismo na região da Mata Atlântica do estado de São Paulo" (Projeto 1). Visando consolidar os produtos turísticos e fortalecer a capacidade de gestão dos parques estaduais paulistas de abrangência do projeto, o projeto adotou medidas como: elaboração de planos de contingência e gerenciamento de riscos, e nova identidade visual para as UCs (gestão); construção/revitalização de infraestrutura, implantação de atrativos, e trilhas adaptadas (infraestrutura); oferecimento de diversos tipos de capacitação e treinamentos para a comunidade, gestores municipais, e micro e pequenos empreendedores (capacitação) (KOGA et al., 2013; SÃO PAULO, 2013). Outro exemplo é o projeto "Consolidação da Gestão do Uso Público nas Unidades de Conservação Estaduais do Estado do Rio de Janeiro" (Projeto 25) que atingiu doze UCs fluminenses, realizando cursos de formação de condutores de visitantes (capacitação); manejo e sinalização de trilhas, implantação de sistema de contagem automática de visitantes (infraestrutura); elaboração de peças de comunicação, implantação de bancos de dados especializados, realização de diagnósticos de potencial turístico, implantação e acompanhamento de planos estratégicos específicos ao uso público (gestão) (PELLEGRINI, 2013).

Tabela 5: Categorias de atuação e quantidade dos projetos de turismo.

Table 5: Acting categories and quantity of tourism projects.

\section{Eixo de atuação}

Gestão

Capacitação

Infraestrutura
Quantidade de projetos

15

4

Em relação aos projetos que contemplam questões relacionadas à gestão do turismo em UCs, estes de modo geral se focam em ações como implantação de roteiros de turismo (Projeto 20) (FUNDAÇÃO SOS MATA ATLÂNTICA, 2018c), criação de mecanismos financeiros que complementem recursos (Projeto 22) (CI BRASIL, [s/d]a), criação de planos municipais de turismo (Projeto 13) (BARTHOLO et al., 2009h; MORAES et al., 2017), e realização de inventários participativos de atrativos (Projeto 2) (WANDEMBRUCK, 2005). Podemos enquadrar tais medidas na categoria "gestão", por compreendê-las como um modo de coordenação que objetiva alcançar metas definidas (EAGLES, 2009). Historicamente, a abordagem de gestão do turismo em áreas protegidas tem sido guiada por um paradigma embasado por uma visão racional e mecanicista, focado em estabelecer objetivos e avaliar de forma técnica alternativas para alcançá-los (PLUMMER; FENNELL, 2009) - o que também foi identificado nos projetos em UCs na Mata Atlântica. Entretanto, tal abordagem pode se revelar como ineficaz para lidar com certas questões complexas que emergem (ARMITAGE et al., 2009). Deste modo, Plummer e Funnell (2009) apontam que estratégias que empreguem uma visão sistêmica e envolvam elementos como comunicação e negociação entre múltiplos atores, tomada de decisão compartilhada, 
e capacidade de adaptação, podem ser mais efetivas para conciliar conservação e desenvolvimento na gestão do turismo em áreas protegidas.

A capacitação como ferramenta de qualificação também foi um elemento-chave identificado. Para além de treinamentos, a construção de habilidades em pessoas atuantes no setor de turismo pode se revelar também como uma forma de empoderamento para comunidades locais (BUTLER, 2017). Um exemplo é o projeto "Formação e organização de Condutores Ambientais Locais pelo IFRS, Campus Porto Alegre" (Projeto 17) que formou 60 condutores ambientais para atuarem em UCs de Porto Alegre e região metropolitana, através do Programa Nacional de Acesso ao Ensino Técnico e Emprego (PRONATEC). Os alunos egressos deste curso formaram a Associação Porto Alegrense de Condutores Ambientais, uma instituição da sociedade civil baseada em princípios da economia solidária. Através de trabalho articulado juntos aos órgãos ambientais estaduais, a Associação propôs uma base legal para regulamentar a atividade, culminando na promulgação de uma Instrução Normativa que estabeleceu normas para a prestação de serviços de turismo em UCs por parte de condutores ambientais autônomos (SILVA et al., 2015). É válido também ressaltar que em muitos casos, a capacitação não deve ocorrer apenas no âmbito da comunidade, como também de outros atores envolvidos, como agentes governamentais (KALIKOSKI et al., 2009). Neste sentido, chamamos a atenção para a importância de projetos em UCs que promovam uma capacitação de múltiplos atores.

A infraestrutura oferecida para o visitante também é um importante fator no turismo em UCs. O número de estruturas como estacionamentos, centros de visitante e mirantes é um dos principais fatores explicativos de atratividade turística para os visitantes em Parques Nacionais no Brasil (VIVEIROS DE CASTRO et al., 2015). Tal informação evidencia a relevância deste componente, embora seja menos relatado nos projetos de turismo aqui investigados (Tabela 5).

Finalmente, é válido salientar que os projetos analisados possuíam informações muito distintas, algumas com certo grau de incerteza. Por exemplo, em relação à conclusão dos projetos, sete já estavam finalizados, sete estavam em andamento, e doze não possuíam informações suficientes para determinar se haviam sido concluídos ou estavam em andamento. De modo geral, nota-se que há uma falta de compilação dos dados de forma sistemática relacionado aos projetos de turismo, o que pode ser um empecilho para um desenvolvimento integrado e sustentável do turismo (BUTLER, 2003). O presente trabalho contribui para preencher esta lacuna de conhecimento.

\section{Considerações finais}

O presente estudo apresentou um panorama dos projetos de turismo em UCs localizadas na Mata Atlântica. A busca direcionada empregando palavras-chaves em ferramentas de busca on-line possibilitou sistematizar as informações disponíveis. A revisão bibliográfica não pretendeu exaurir o tema, mas sim organizar e analisar de maneira sistemática tais informações frente a literatura científica e cinza existente.

De modo geral, os projetos se caracterizaram por serem intervenções de atores não governamentais, e ocorrerem principalmente na região sudeste. A categoria de UCs em que mais se desenvolveram projetos de turismo foram os Parques, e muitos projetos possuem financiamento advindo de verbas públicas. Além disso, o envolvimento de ONGs, OSCIPs e associações comunitárias se destaca no 
planejamento e/ou execução destes. Os projetos atuam em diversas frentes, visando melhorar aspectos relacionados à gestão, capacitação dos atores sociais e/ou infraestrutura das UCs. Apresentar tais aspectos dos projetos analisados permitiu identificar como o turismo tem sido fomentado, podendo abrir caminhos para futuros estudos e iniciativas. Finalmente, esta pesquisa possui potencial de aplicação na gestão de UCs, ao expor como intervenções vem sendo feitas para estimular a prática do ecoturismo no recorte específico da Mata Atlântica.

\section{Referências}

ADAMS, C.; MOTTA, R. S.; ORTIZ, R. A.; REID, J., AZNAR, C., E.; SINISGALLI, P. $A$. The use of contingent valuation for evaluating protected areas in the developing world: Economic valuation of Morro do Diabo State Park, Atlantic Rainforest, São Paulo State (Brazil). Ecological Economics, v. 66, p. 359-370, 2008.

ARMitAGE, D. R.; PlUMMER, R.; BERKES, F.; ARTHUR, R. I.; CHARLES, A. T., DAVIDSON-HUNT, I. J., DIDUCK, A. P.; DOUBLEDAY, N. C.; JOHNSON, D. S.; MARSCHKE, M.; MCCONNEY, P.; PINKERTON, E. W.; WOLLENBERG, E. K. Adaptive Co-Management for Social-Ecological Complexity. Frontiers in Ecology and the Environment, v. 7, n. 2, 95-102, 2009.

ASSOCIAÇÃO MICO-LEÃO DOURADO. Ecoturismo. [s/d]. Disponível em: $<$ http://www.micoleao.org.br/index.php > Acesso em: 14/11/2019.

BARTHOLO, R.; SANSOLO, D. G.; BURSZTYN, I. Tramas em Riacho Doce Turismo de base comunitária: olhares e experiências brasileiras. Letra e Imagem, p. 398-399, 2009a.

BARTHOLO, R.; SANSOLO, D. G.; BURSZTYN, I. Base Local Ecoturismo: promovendo o turismo de base comunitária na Costa do Cacau-BA - Turismo de base comunitária: olhares e experiências brasileiras. Letra e Imagem, p. 403-405, 2009b.

BARTHOLO, R.; SANSOLO, D. G.; BURSZTYN, I. Estruturação e fortalecimento do turismo de base comunitária do povoado Terra Caída - Turismo de base comunitária: olhares e experiências brasileiras. Letra e Imagem, p. 430-431, 2009c.

BARTHOLO, R.; SANSOLO, D. G.; BURSZTYN, I. Ecobase Ilha das Caieiras Turismo de base comunitária: olhares e experiências brasileiras. Letra e Imagem, p. 438-439, 2009d.

BARTHOLO, R.; SANSOLO, D. G.; BURSZTYN, I. Ecoturismo de base comunitária da região da Trilha do Ouro - Turismo de base comunitária: olhares e experiências brasileiras. Letra e Imagem, p. 452-453, 2009 e.

BARTHOLO, R.; SANSOLO, D. G.; BURSZTYN, I. Turismo sustentável de base comunitária na Reserva de Desenvolvimento Sustentável da Barra do Una Turismo de base comunitária: olhares e experiências brasileiras. Letra e Imagem, p. 474-475, $2009 f$.

BARTHOLO, R.; SANSOLO, D. G.; BURSZTYN, I. Deslocamentos: ecoturismo de base comunitária no litoral norte do Paraná - Turismo de base comunitária: olhares e experiências brasileiras. Letra e Imagem, p. 476-478, 2009g.

BARTHOLO, R.; SANSOLO, D. G.; BURSZTYN, I. Boas práticas para o turismo comunitário - Turismo de base comunitária: olhares e experiências brasileiras. Letra e Imagem, p. 476-478, 2009h. 
BRASIL. Ministério do Meio Ambiente (MMA). Corredores ecológicos: experiências em planejamento e implementação. 2007. Disponível em $<$ http://www.meioambiente.ba.gov.br/arquivos/File/Publicacoes/Cadernos/CE Experi encias.pdf> Acesso em: 14/11/2019.

BRASIL. Ministério do Meio Ambiente (MMA). Diagnóstico da visitação em parques nacionais e estaduais. 2009 . Disponível em: $<$ https://www.mma.gov.br/estruturas/sbs dap/ arquivos/diagnostico da visitacao e m parques.pdf > Acesso em: 14/11/2019.

BRASIL. Ministério do Meio Ambiente (MMA). Painel Unidades de Conservação Brasileiras. 2020. Disponível em: <https://bit.ly/3a6DjIC> Acesso em: 11/02/2020.

BRASIL. Ministério do Turismo (MTur). Ecoturismo: Orientações básicas. 2010. Disponível em: $<$ http://www.turismo.gov.br/sites/default/turismo/o ministerio/publicacoes/downloads publicacoes/Ecoturismo Versxo Final IMPRESSxO .pdf> Acesso em: 14/11/2019. BRATMAN, G. N.; ANDERSON, C. B.; BERMAN, M. G.; COCHRAN, B.; DE VRIES, S.; FLANDERS, J.; FOLKE, C.; FRUMKIN, H.; GROSS, J. J.; HARTIG, T.; KAHN, P. H.; KUO, M.; LAWLER, J. J.; LEVIN, P. S.; LINDAHL, T.; MEYER-LINDENBERG, A.; MITCHELL, R.; OUYANG, Z.; ROE, J., L.; SCARLETT, L.; SMITH, J. R.; VAN DEN BOSCH, M.; WHEELER, B.W.; WHITE, M. P.; ZHENG, H.; DAILY, G. C. Nature and mental health: An ecosystem service perspective. Science Advances, v. 5, n. 7, 2019.

BROWN, L. D. Bridging Organizations and Sustainable Development. Human Relations, v. 44, n. 8, 1991.

BUTLER, G. Fostering community empowerment and capacity building through tourism: perspectives from Dullstroom, South Africa. Journal of Tourism and Cultural Change, v. 15, n. 3, 199-212, 2017.

BUTLER, R. W. Problems and Issues of Integrating Tourism Development. Contemporary Issues in Tourism Development. In: BUTLER, R. W.; PEARCE, D. G. (org.) Tourism development. London: Routledge, p. 65-80, 2003.

CAMINHO DA MATA ATLÂNTICA. O que é o Caminho da Mata Atlântica? [s/d]. Disponível em: <http://caminhodamataatlantica.org.br/o-que-e-o-caminho-da-mataatlantica/> Acesso em: 14/11/2019.

CONSERVAÇÃO INTERNACIONAL BRASIL. Potencializando o uso público das Unidades de Conservação. $[\mathrm{s} / \mathrm{d}] \mathrm{a}$ Disponível em: $<$ https://www.conservation.org/brasil/iniciativas-atuais/potencializando-o-uso-dasunidades-de-conservacao > Acesso em: 14/11/2019.

CONSERVAÇÃO INTERNACIONAL BRASIL. Projeto de fortalecimento do Parque Nacional do Pau-Brasil e entorno. [s/d]b. Disponível em: $<$ https://www.conservation.org/brasil/iniciativas-atuais/projeto-de-fortalecimento-doparque-nacional-do-pau-brasil-e-entorno> Acesso em: 14/11/2019.

COSTA, C. M. M. A evolução nas publicações sobre turismo de natureza nas revistas brasileiras de turismo: uma análise cienciométrica. 2015. Trabalho de conclusão de curso (Bacharelado em turismo) - Centro de Excelência em Turismo, Universidade de Brasília, Brasília, 2015. 
COSTA, G. V. L.; CATÃO, H.; PRADO, R. M. Praia do Aventureiro: um caso sui generis de gestão local do turismo. In: BARTHOLO, R.; SANSOLO, D. G.; BURSZTYN, I. (orgs.) Turismo de base comunitária: olhares e experiências brasileiras. Letra e Imagem, p. 177-197, 2009.

CRONA, B. I.; PARKER, J. N. Learning in support of governance: Theories, methods, and a framework to assess how bridging organizations contribute to adaptive resource governance. Ecology and Society, v. 17, n. 1, 2012.

DHARMARATNE, G. S.; SANG, F. Y.; WALLING, L. J. Tourism potentials for financing protected areas. Annals of Tourism Research, v. 27, n. 3, p. 590-610, 2000.

DIAS, A. C. E. Guia: como elaborar uma revisão bibliográfica. Instituto Nacional de Pesquisas Espaciais (INPE). 2016. Disponível em: <http://urlib.net/8JMKD3MGP3W34P/3MB5C7H> Acesso em: 14/11/2019.

DÍAZ, S.; PASCUAL, U.; STENSEKE, M. et al. (+ 27 autores) Assessing nature's contributions to people. Science, v. 359, n. 6373, p. 270-272, 2017.

DINIZ, I. N. Projeto quer criar megatrilha de $3.000 \mathrm{~km}$ pela mata atlântica. Folha de São Paulo, ano 98, n. 32527, 23 abril 2018. Disponível em: $<$ https://www1.folha.uol.com.br/ambiente/2018/04/projeto-quer-criar-megatrilha-de3000-km-pela-mata-atlantica.shtml> Acesso em: 14/11/2019.

EAGLES, P. F. J. Governance of recreation and tourism partnerships in parks and protected areas. Journal of Sustainable Tourism, v. 17, n. 2, p. 231-248, 2009.

FERREIRA, H. C. H. Turismo comunitário, tradicionalidade e reserva de desenvolvimento sustentável na defesa do território nativo: Aventureiro-Ilha Grande/RJ. Revista Brasileira de Pesquisa em Turismo, v. 8, n. 2, p. 361-379, 2014.

FUNDAÇÃO SOS MATA ATLÂNTICA. Unidades de Conservação da Costa de Araranguá - difundindo a natureza local com o Roteiro Geoecológico. 2018a. Disponível em: <https://www.sosma.org.br/107032/conheca-os-projetos-que-seraoapoiados-por-edital-que-beneficia-unidades-de-conservacao/> Acesso em: 14/11/2019.

FUNDAÇÃO SOS MATA ATLÂNTICA. Programa de abertura de escalada em rocha na Floresta Nacional de Ipanema, e monitoramento do Urubu-Rei nos setores de escalada. 2018b. Disponível em: <https://www.sosma.org.br/107032/conheca-osprojetos-que-serao-apoiados-por-edital-que-beneficia-unidades-de-conservacao/> Acesso em: 14/11/2019.

FUNDAÇÃO SOS MATA ATLÂNTICA. Trilhas da Gralha Azul - Projeto de Turismo de Base Comunitária com o protagonismo juvenil na Flona do Assungui Campo Largo (PR). 2018c. Disponível em: $<$ https://www.sosma.org.br/107032/conheca-os-projetos-que-serao-apoiados-poredital-que-beneficia-unidades-de-conservacao/> Acesso em: 14/11/2019.

ICMBio - Instituto Chico Mendes de Conservação da Biodiversidade. Painel Dinâmico de Informações. 2019. Disponível em: <http://www.qv.icmbio.gov.br> Acesso em: 14/11/2019.

ICMBio - Instituto Chico Mendes de Conservação da Biodiversidade. Aviso: COVID19 - Suspensão de visitação pública. 2020. Disponível em: <https://www.icmbio.gov.br/portal/ultimas-noticias/20-geral/10962-aviso-covid-19suspencao-de-visitacao-publica> Acesso em: 24/10/2020. 
INSTITUTO TERRA DE PRESERVAÇÃO AMBIENTAL (ITPA). Gestão do uso público nos parques estaduais do Rio de Janeiro. [s/d] Disponível em: $<$ http://www.itpa.org.br/?page id=2427> Acesso em: 14/11/2019.

JAMAL, T.; KREUTER, U.; YANOSKY, A. Bridging organisations for sustainable development and conservation: a Paraguayan case. Int. J. Tourism Policy, v. 1, n. 2, p. 93-110, 2007.

JAMAL, T.; STRONZA, A. Collaboration theory and tourism practice in protected areas: Stakeholders, structuring and sustainability. Journal of Sustainable Tourism, v. 17, n. 2, p. 169-189, 2009.

KALIKOSKI, D. C.; SEIXAS, C. S.; ALMUDI, T. Gestão compartilhada e comunitária da pesca no Brasil: Avanços e desafios. Ambiente e Sociedade, v. 12, n. 1, p. 151172, 2009.

KOGA, E. S.; OLIVEIRA, C.S.; KANESHIRO, D.M. Programa de capacitação do Projeto de Ecoturismo na Mata Atlântica no entorno dos parques estaduais paulistas. Revista Brasileira de Ecoturismo, v.6, n.1, p.255-268, 2013.

LOPES, L. Projeto leva jovens para interagir e preservar reservas ambientais. Jornal da USP, 13 junho 2017. Disponível em: <http://www.jornal.usp.br/?p=91386> Acesso em: 14/11/2019.

LUO, Y.; DENG, J. The new environmental paradigm and nature-based tourism motivation. Journal of Travel Research, v. 46, p. 392-402, 2008.

MENDONÇA, T. C. M. Turismo socialmente responsável da Prainha do Canto Verde: uma solução em defesa do local herdado. In: BARTHOLO, R.; SANSOLO, D. G.; BURSZTYN, I. (orgs.) Turismo de base comunitária: olhares e experiências brasileiras. Letra e Imagem, p. 289-301, 2009.

MORAES, E. A.; MENDONÇA, T. C. M.; PINHEIRO, C. V. Trilhando o turismo de base comunitária em Minas: um novo caminho das Gerais. CULTUR, v. 11, n. 1, 2017.

NEIMAN, Z.; SARACENI, R. F.; GEERDINK, S. Levantamento quali-quantitativo da produção científica sobre Ecoturismo no Brasil. Revista Brasileira de Ecoturismo, v. 3, n. 3, p. $528-555,2010$.

PELLEGRINI, F. Explorando o uso público em Unidades de Conservação. O Eco, 04 julho 2013. Disponível em: <https://www.oeco.org.br/reportagens/27346-explorandoo-uso-publico-em-unidade-de-conservacao/> Acesso em: 14/11/2019.

PLUMMER, R.; FENNELL, D. A. Managing protected areas for sustainable tourism: Prospects for adaptive co-management. Journal of Sustainable Tourism, v. 17, n. 2, p. 149-168, 2009.

PRADO, D. S. Processos de cogestão e seus avanços em reservas extrativistas costeiras e marinhas do Brasil. 2019. Tese (Doutorado em Ambiente e Sociedade) Núcleo de Estudos e Pesquisas Ambientais da Universidade Estadual de Campinas. Campinas, 2019.

PRADO, D. S.; SEIXAS, C. S.; BERKES, F. Looking back and looking forward: Exploring livelihood change and resilience building in a Brazilian coastal community.

Ocean and Coastal Management, v. 113, p. 29-37, 2015. 
PREFEITURA DE ITU - SECRETARIA DE MEIO AMBIENTE. Plano municipal de conservação e recuperação da Mata Atlântica. 2017. Disponível em: $<$ https://itu.sp.gov.br/meio-ambiente/programa-municipio-verde-azul/planos-eprogramas/> Acesso em: 14/11/2019.

REZENDE, C. L.; SCARANO, F. R.; ASSAD, E. D.; JOLY, C. A.; METZGER, J. P.; STRASSBURG, B. B. N.; TABARELLI, M.; FONSECA, G. A.; MITTERMEIER, R. A. From hotspot to hopespot: An opportunity for the Brazilian Atlantic Forest. Perspectives in Ecology and Conservation, v. 16, n. 4, p. 208-214, 2018.

RODRIGUES, C. G. de O.; GODOY, L. R. da C. Atuação pública e privada na gestão de Unidades de Conservação: aspectos socioeconômicos da prestação de serviços de apoio à visitação em parques nacionais. Desenvolvimento e Meio Ambiente, v. 28, p. 75-88, 2013.

RODRIGUES, S. Governo corta R $\$ 187$ milhões do MMA. Saiba como o corte foi dividido. OEco, 07 maio 2019. Disponível em: < https://www.oeco.org.br/noticias/governo-corta-r-187-milhoes-do-mma-saiba-como-ocorte-foi-dividido/> Acesso em: 11/02/2020.

SALVADOR, M. C. C.; PEDROSO, R.; BASTOS, F. B. Projeto de turismo de base comunitária: comunidade tradicional do Bonete, Ilhabela (SP). Revista Brasileira de Ecoturismo, São Paulo, v.6, n.5, p.1052-1069, 2013.

SÃO PAULO - GOVERNO DO ESTADO DE SÃO PAULO, SECRETARIA DO MEIO AMBIENTE. Projeto de Desenvolvimento do Ecoturismo na Região da Mata Atlântica no Estado de São Paulo. São Paulo, SMA: 2013.

SILVA, C. R. C.; CUNHA, A. M.; BAZOTTI, L. S.; NASCIMENTO, C. A. Formação e organização de condutores ambientais locais: estratégias de desenvolvimento do turismo sustentável em Unidades de Conservação gaúchas. 2015. In: HANAZAKI, N.; HERBST, D. F.; AVILA, J. V. C.; HEINEBERG, M. R.; GOMES, T. C. C. (Orgs.) Anais do VII Seminário Brasileiros sobre Áreas Protegidas e Inclusão Social e II Encontro Latino Americano sobre Áreas Protegidas e Inclusão Social. Florianópolis: Universidade Federal de Santa Catarina, 2015. p. 211-219.

SOUZA, T. V. S. B.; SIMÕES, H. B. Contribuições do Turismo em Unidades de Conservação Federais para a Economia Brasileira - Efeitos dos Gastos dos Visitantes em 2017: Sumário Executivo. ICMBio. Brasília. 2018.

SOUZA, T. V. S. B.; THAPA, B. Tourism Demand Analysis of the Federal Protected Areas of Brazil. Journal of Park and Recreation Administration, v. 36, p. 1-21, 2018.

SPINOLA, C. A. O ecoturismo, o desenvolvimento local e a conservação da natureza em espaços naturais protegidos: objetivos conflitantes? RDE - Revista de Desenvolvimento Econômico, ano VIII, n. 13, 2006.

SPVS - Sociedade de Pesquisa em Vida Selvagem e Educação Ambiental. Relatório Anual 2011. 2011. Disponível em: <http://www.spvs.org.br/quem-somos/relatoriosanuais/> Acesso em: 14/11/2019.

UNWTO - WORLD TOURISM ORGANIZATION. International Tourism Highlights. 2019. Disponível em: <https://www.e-unwto.org/doi/pdf/10.18111/9789284421152> Acesso em: 14/11/2019.

VIVEIROS DE CASTRO, E. V.; SOUZA, T. B.; THAPA, B. Determinants of tourism attractiveness in the national parks of Brazil. Parks, v. 21, n. 2, p. 51-62, 2015. 
WANDEMBRUCK, A. 2005. Planejamento do ecoturismo participativo - um estudo de caso na Vila das Peças, Guaraqueçaba, Paraná, Brasil. Dissertação (Mestrado em Ciências Florestais) - Setor de Ciências Agrárias, Universidade Federal do Paraná, Curitiba, 2005.

WHITELAW, P. A.; KING, B. E. M.; TOLKACH, D. Protected areas, conservation and tourism - financing the sustainable dream. Journal of Sustainable Tourism, v. 22, n. 4, p. 584-603, 2014.

WILSON, E.; NIELSEN, N.; BUULTJENS, J. From lessees to partners: Exploring tourism public-private partnerships within the New South Wales national parks and wildlife service. Journal of Sustainable Tourism, v. 17, n. 2, p. 269-285, 2009.

WWF Brasil. Manual de ecoturismo de base comunitária: ferramentas para um planejamento responsável. WWF Brasil: Brasília. 2003. 470p.

\section{Agradecimentos}

Os autores agradecem ao Programa Institucional de Bolsas de Iniciação Científica da Universidade Estadual de Campinas pela concessão da bolsa ao primeiro autor, e aos colegas do Laboratório de Ecologia e Manejo de Ecossistemas (LEME) e do grupo de pesquisa Conservação e Gestão de Recursos Naturais de Uso Comum (CGCommons), em especial Fernanda Watanabe e Sandrine Gouvêa.

Rafael Cavalcanti Lembi: Universidade Estadual de Campinas, Campinas, SP, Brasil.

E-mail: rclembi@gmail.com

Link para o currículo Lattes: http://lattes.cnpq.br/2549378970906577

Leandra Regina Gonçalves: Universidade Federal de São Paulo, Santos, SP, Brasil.

E-mail: goncalves.leandra@unifesp.br

Link para o currículo Lattes: http://lattes.cnpq.br/8461942312318325

Cristiana Simão Seixas: Universidade Estadual de Campinas, Campinas, SP, Brasil.

E-mail: csseixas@unicamp.br

Link para o currículo Lattes: http://lattes.cnpq.br/8835529370621973

Data de submissão: 25 de fevereiro de 2021

Data de recebimento de correções: 08 de outubro de 2021

Data do aceite: 08 de outubro de 2021

Avaliado anonimamente 
APÊNDICE 1: Compilação dos projetos de turismo em Unidades de Conservação na Mata Atlântica identificados através do levantamento bibliográfico.

APPENDIX 1: Compilation of tourism projects in protected areas in the Atlantic Forest that were identified through the bibliographical survey.

\begin{tabular}{|c|c|c|c|c|c|c|}
\hline & Projeto & $\begin{array}{l}\text { Unidade(s) de } \\
\text { Conservação de } \\
\text { abrangência }\end{array}$ & Financiamento & $\begin{array}{c}\text { Principais tipos de } \\
\text { organizações envolvidas }\end{array}$ & $\begin{array}{l}\text { Eixos de } \\
\text { atuação }\end{array}$ & Referências \\
\hline 1 & $\begin{array}{l}\text { Projeto de } \\
\text { Desenvolvimento do } \\
\text { Ecoturismo na região } \\
\text { da Mata Atlântica no } \\
\text { Estado de São Paulo }\end{array}$ & $\begin{array}{l}\text { Parques Estaduais: } \\
\text { "Carlos Botelho", } \\
\text { Intervales, Caverna } \\
\text { do Diabo, Turístico } \\
\text { Alto Ribeira } \\
\text { (PETAR), Ilha do } \\
\text { Cardoso e llhabela } \\
\text { (SP) }\end{array}$ & $\begin{array}{l}\text { Governo Estadual+Banco } \\
\text { internacional }\end{array}$ & Órgãos governamentais & $\begin{array}{l}\text { Capacitação } \\
\text { Gestão } \\
\text { Infraestrutura }\end{array}$ & $\begin{array}{l}\text { KOGA et al. (2013), } \\
\text { SÃO PAULO (2013) }\end{array}$ \\
\hline 2 & $\begin{array}{l}\text { Programa de } \\
\text { Ecoturismo para a Vila } \\
\text { das Peças }\end{array}$ & $\begin{array}{l}\text { Área de Proteção } \\
\text { Ambiental Federal } \\
\text { de Guaraqueçaba } \\
\text { (PR) }\end{array}$ & $\begin{array}{l}\text { Governo } \\
\text { Federal+Organização da } \\
\text { Sociedade Civil }\end{array}$ & $\begin{array}{l}\text { Associação comunitária, } \\
\text { Universidades/Institutos }\end{array}$ & $\begin{array}{l}\text { Capacitação } \\
\text { Gestão }\end{array}$ & $\begin{array}{l}\text { WANDEMBRUCK } \\
\text { (2005) }\end{array}$ \\
\hline 3 & $\begin{array}{l}\text { Projeto Turismo de } \\
\text { Base Comunitária }\end{array}$ & $\begin{array}{l}\text { área de influência do } \\
\text { Parque Estadual } \\
\text { Ilhabela (SP) }\end{array}$ & - & $\begin{array}{l}\text { Empresa, ONGs/OSCIPs, } \\
\text { Universidades/Institutos }\end{array}$ & $\begin{array}{l}\text { Capacitação } \\
\text { Gestão }\end{array}$ & $\begin{array}{l}\text { SALVADOR et al. } \\
(2013)\end{array}$ \\
\hline 4 & $\begin{array}{l}\text { Projeto de Extensão } \\
\text { Povo do Aventureiro: } \\
\text { fortalecimento do } \\
\text { turismo de base } \\
\text { comunitária }\end{array}$ & $\begin{array}{l}\text { Reserva de } \\
\text { Desenvolvimento } \\
\text { Sustentável } \\
\text { Estadual do } \\
\text { Aventureiro (RJ) }\end{array}$ & Governo Federal & $\begin{array}{l}\text { Associação comunitária } \\
\text { ONGs/OSCIPs, } \\
\text { Universidades/Institutos }\end{array}$ & Gestão & $\begin{array}{l}\text { COSTA et al. (2009), } \\
\text { FERREIRA (2014) }\end{array}$ \\
\hline 5 & $\begin{array}{l}\text { Projeto Turístico } \\
\text { Socialmente } \\
\text { Responsável }\end{array}$ & $\begin{array}{l}\text { Reserva Extrativista } \\
\text { Federal Prainha do } \\
\text { Canto Verde (CE) }\end{array}$ & Comunitário & Associação comunitária & Gestão & MENDONÇA (2009) \\
\hline 6 & $\begin{array}{l}\text { Tramas em Riacho } \\
\text { Doce }\end{array}$ & $\begin{array}{l}\text { Área de Proteção } \\
\text { Ambiental Federal } \\
\text { Costa dos Corais } \\
\text { (AL, PE) }\end{array}$ & Governo Federal & $\begin{array}{l}\text { Associação comunitária, } \\
\text { ONGs/OSCIPs }\end{array}$ & Capacitação & $\begin{array}{l}\text { BARTHOLO et al. } \\
(2009 a)\end{array}$ \\
\hline 7 & $\begin{array}{l}\text { Base Local Turismo: } \\
\text { promovendo o turismo } \\
\text { de base comunitária na } \\
\text { Costa do Cacau, BA }\end{array}$ & $\begin{array}{l}\text { Áreas de Proteção } \\
\text { Ambiental } \\
\text { Estaduais: Costa de } \\
\text { Itacaré; Lagoa } \\
\text { Encantada (BA) }\end{array}$ & Governo Federal & $\begin{array}{l}\text { Associação comunitária, } \\
\text { ONGs/OSCIPs }\end{array}$ & Capacitação & $\begin{array}{l}\text { BARTHOLO et al. } \\
(2009 b)\end{array}$ \\
\hline
\end{tabular}




\begin{tabular}{|c|c|c|c|c|c|c|}
\hline & Projeto & $\begin{array}{l}\text { Unidade(s) de } \\
\text { Conservação de } \\
\text { abrangência }\end{array}$ & Financiamento & $\begin{array}{c}\text { Principais tipos de } \\
\text { organizações envolvidas }\end{array}$ & $\begin{array}{l}\text { Eixos de } \\
\text { atuação }\end{array}$ & Referências \\
\hline 8 & $\begin{array}{l}\text { Estruturação e } \\
\text { fortalecimento do } \\
\text { turismo de base } \\
\text { comunitária do } \\
\text { povoado de Terra } \\
\text { Caída }\end{array}$ & $\begin{array}{l}\text { Área de Proteção } \\
\text { Ambiental Estadual } \\
\text { Litoral Sul (SE) }\end{array}$ & Governo Federal & $\begin{array}{l}\text { Associação comunitária, } \\
\text { ONGs/OSCIPs }\end{array}$ & Gestão & $\begin{array}{l}\text { BARTHOLO et al. } \\
(2009 c)\end{array}$ \\
\hline 9 & $\begin{array}{l}\text { Ecobase Ilha das } \\
\text { Caieiras }\end{array}$ & $\begin{array}{l}\text { Estação Ecológica } \\
\text { Municipal do } \\
\text { Lameirão e Parque } \\
\text { Natural Municipal } \\
\text { Baía Noroeste (ES) }\end{array}$ & Governo Federal & $\begin{array}{l}\text { Órgãos governamentais, } \\
\text { ONGs/OSCIPs }\end{array}$ & Gestão & $\begin{array}{l}\text { BARTHOLO et al. } \\
\text { (2009d) }\end{array}$ \\
\hline 10 & $\begin{array}{l}\text { Ecoturismo de base } \\
\text { comunitária da região } \\
\text { da Trilha do Ouro }\end{array}$ & $\begin{array}{l}\text { Parque Nacional da } \\
\text { Serra da Bocaina } \\
(\mathrm{RJ}, \mathrm{SP})\end{array}$ & Governo Federal & ONGs/OSCIPs & $\begin{array}{l}\text { Capacitação } \\
\text { Gestão }\end{array}$ & $\begin{array}{l}\text { BARTHOLO et al. } \\
(2009 e)\end{array}$ \\
\hline 11 & $\begin{array}{l}\text { Turismo sustentável de } \\
\text { base comunitária na } \\
\text { Reserva de } \\
\text { Desenvolvimento } \\
\text { Sustentável Barra do } \\
\text { Uma }\end{array}$ & $\begin{array}{l}\text { Reserva de } \\
\text { Desenvolvimento } \\
\text { Sustentável } \\
\text { Estadual Barra do } \\
\text { Una (SP) }\end{array}$ & Governo Federal & Associação comunitária & Gestão & $\begin{array}{l}\text { BARTHOLO et al. } \\
\text { (2009f) }\end{array}$ \\
\hline 12 & $\begin{array}{l}\text { Deslocamentos: } \\
\text { ecoturismo de base } \\
\text { comunitária no litoral } \\
\text { norte do Paraná }\end{array}$ & $\begin{array}{l}\text { Área de Proteção } \\
\text { Ambiental Federal } \\
\text { Guaraqueçaba (PR) }\end{array}$ & Governo Federal & ONGs/OSCIPs & $\begin{array}{l}\text { Capacitação } \\
\text { Gestão }\end{array}$ & $\begin{array}{l}\text { BARTHOLO et al. } \\
(2009 \mathrm{~g})\end{array}$ \\
\hline 13 & $\begin{array}{l}\text { Boas práticas de } \\
\text { turismo de base } \\
\text { comunitária no } \\
\text { Território da Serra do } \\
\text { Brigadeiro }\end{array}$ & $\begin{array}{l}\text { área de influência do } \\
\text { Parque Estadual da } \\
\text { Serra do Brigadeiro } \\
\text { (MG) }\end{array}$ & Governo Federal & Associação comunitária & $\begin{array}{l}\text { Capacitação } \\
\text { Gestão }\end{array}$ & $\begin{array}{l}\text { MORAES et al. } \\
\text { (2017); BARTHOLO } \\
\text { et al. (2009h) }\end{array}$ \\
\hline 14 & $\begin{array}{l}\text { Plano municipal de } \\
\text { conservação e } \\
\text { recuperação da Mata } \\
\text { Atlântica do município } \\
\text { de ltu }\end{array}$ & $\begin{array}{l}\text { áreas de Proteção } \\
\text { Ambiental } \\
\text { municipais de Itu } \\
\text { (SP) }\end{array}$ & - & Órgãos governamentais & $\begin{array}{l}\text { Capacitação } \\
\text { Gestão } \\
\text { Infraestrutura }\end{array}$ & $\begin{array}{l}\text { PREFEITURA DE } \\
\text { ITU (2017) }\end{array}$ \\
\hline
\end{tabular}


Lembi, R.C.; Gonçalves, L.R.; Seixas, C.S.

\begin{tabular}{|c|c|c|c|c|c|c|}
\hline & Projeto & $\begin{array}{l}\text { Unidade(s) de } \\
\text { Conservação de } \\
\text { abrangência }\end{array}$ & Financiamento & $\begin{array}{c}\text { Principais tipos de } \\
\text { organizações envolvidas }\end{array}$ & $\begin{array}{l}\text { Eixos de } \\
\text { atuação }\end{array}$ & Referências \\
\hline 15 & $\begin{array}{l}\text { Cooperaguá e o } \\
\text { ecoturismo na APA de } \\
\text { Guaraqueçaba }\end{array}$ & $\begin{array}{l}\text { Área de Proteção } \\
\text { Ambiental } \\
\text { Guaraqueçaba (PR) }\end{array}$ & $\begin{array}{l}\text { Organização da } \\
\text { Sociedade Civil de } \\
\text { Interesse Público }\end{array}$ & $\begin{array}{l}\text { ONGs/OSCIPs, Associação } \\
\text { Comunitária }\end{array}$ & $\begin{array}{l}\text { Gestão } \\
\text { Infraestrutura }\end{array}$ & SPVS (2011) \\
\hline 16 & $\begin{array}{l}\text { Turismo sustentável } \\
\text { nos corredores } \\
\text { ecológicos do estado } \\
\text { do Espírito Santo }\end{array}$ & $\begin{array}{l}\text { Reserva Biológica } \\
\text { Federal de } \\
\text { Comboios, Floresta } \\
\text { Nacional de } \\
\text { Goitacazes, Parque } \\
\text { Nacional do } \\
\text { Caparaó, Parque } \\
\text { Estadual de Itaúnas } \\
\text { (ES) }\end{array}$ & $\begin{array}{l}\text { Governo Federal+Banco } \\
\text { internacional }\end{array}$ & Órgãos governamentais & Capacitação & BRASIL (2007) \\
\hline 17 & $\begin{array}{l}\text { Formação e } \\
\text { organização de } \\
\text { Condutores Ambientais } \\
\text { Locais pelo IFRS, } \\
\text { Campus Porto Alegre }\end{array}$ & $\begin{array}{l}\text { Parque Estadual de } \\
\text { Itapuã (RS) }\end{array}$ & Governo Federal & Universidades/Institutos & $\begin{array}{l}\text { Capacitação } \\
\text { Gestão }\end{array}$ & SILVA et al. (2015) \\
\hline 18 & $\begin{array}{l}\text { Unidades de } \\
\text { Conservação da Costa } \\
\text { de Araranguá - } \\
\text { difundindo a natureza } \\
\text { local com o Roteiro } \\
\text { Geoecológico }\end{array}$ & $\begin{array}{l}\text { Unidades de } \\
\text { Conservação em } \\
\text { Araranguá (SC) }\end{array}$ & $\begin{array}{l}\text { Organização da } \\
\text { Sociedade Civil de } \\
\text { Interesse } \\
\text { Público+Empresa privada }\end{array}$ & Empresa & Gestão & $\begin{array}{l}\text { FUNDAÇÃO SOS } \\
\text { MATA ATLÂNTICA } \\
(2018 a)\end{array}$ \\
\hline 19 & $\begin{array}{l}\text { Programa de abertura } \\
\text { de escalada em rocha } \\
\text { na Floresta Nacional } \\
\text { de Ipanema, e } \\
\text { monitoramento do } \\
\text { Urubu-Rei nos setores } \\
\text { de escalada }\end{array}$ & $\begin{array}{l}\text { Floresta Nacional de } \\
\text { Ipanema (SP) }\end{array}$ & $\begin{array}{l}\text { Organização da } \\
\text { Sociedade Civil de } \\
\text { Interesse } \\
\text { Público+Empresa privada }\end{array}$ & ONGs/OSCIPs & $\begin{array}{l}\text { Capacitação } \\
\text { Gestão }\end{array}$ & $\begin{array}{l}\text { FUNDAÇÃO SOS } \\
\text { MATA ATLÂNTICA } \\
(2018 b)\end{array}$ \\
\hline
\end{tabular}




\begin{tabular}{|c|c|c|c|c|c|c|}
\hline & Projeto & $\begin{array}{l}\text { Unidade(s) de } \\
\text { Conservação de } \\
\text { abrangência }\end{array}$ & Financiamento & $\begin{array}{c}\text { Principais tipos de } \\
\text { organizações envolvidas }\end{array}$ & $\begin{array}{l}\text { Eixos de } \\
\text { atuação }\end{array}$ & Referências \\
\hline 20 & $\begin{array}{l}\text { Trilhas da Gralha Azul } \\
\text { - Projeto de Turismo } \\
\text { de Base Comunitária } \\
\text { com o protagonismo } \\
\text { juvenil na Flona do } \\
\text { Assungui - Campo } \\
\text { Largo (PR) }\end{array}$ & $\begin{array}{l}\text { Floresta Nacional de } \\
\text { Assungui (PR) }\end{array}$ & $\begin{array}{l}\text { Organização da } \\
\text { Sociedade Civil de } \\
\text { Interesse } \\
\text { Público+Empresa privada }\end{array}$ & ONGs/OSCIPs & $\begin{array}{l}\text { Capacitação } \\
\text { Gestão }\end{array}$ & $\begin{array}{l}\text { FUNDAÇÃO SOS } \\
\text { MATA ATLÂNTICA } \\
(2018 \mathrm{c})\end{array}$ \\
\hline 21 & $\begin{array}{l}\text { Caminho da Mata } \\
\text { Atlântica }\end{array}$ & $\begin{array}{l}\text { mais de } 70 \text { UCs } \\
\text { (PR, RJ, SC, SP, } \\
\text { RS) }\end{array}$ & - & ONGs/OSCIPs & Gestão & $\begin{array}{l}\text { DINIZ (2018); } \\
\text { CAMINHO DA MATA } \\
\text { ATLÂNTICA ([s/d]) }\end{array}$ \\
\hline 22 & $\begin{array}{l}\text { Potencializando o uso } \\
\text { público das Unidades } \\
\text { de Conservação }\end{array}$ & $\begin{array}{l}\text { UCs do território de } \\
\text { Abrolhos (BA) }\end{array}$ & - & ONGs/OSCIPs & Gestão & $\begin{array}{l}\text { CONSERVAÇÃO } \\
\text { INTERNACIONAL } \\
\text { BRASIL ([s/d]a) }\end{array}$ \\
\hline 23 & $\begin{array}{l}\text { Projeto de } \\
\text { Fortalecimento do } \\
\text { Parque Nacional do } \\
\text { Pau-Brasil e entorno }\end{array}$ & $\begin{array}{l}\text { Parque Nacional do } \\
\text { Pau Brasil (BA) }\end{array}$ & - & ONGs/OSCIPs & Gestão & $\begin{array}{l}\text { CONSERVAÇẤO } \\
\text { INTERNACIONAL } \\
\text { BRASIL ([s/d]b) }\end{array}$ \\
\hline 24 & $\begin{array}{l}\text { Programa de } \\
\text { Ecoturismo e } \\
\text { Conservação Na Trilha } \\
\text { do Mico-Leão-Dourado }\end{array}$ & $\begin{array}{l}\text { Reserva Biológica } \\
\text { Federal de Poço das } \\
\text { Antas (RJ) }\end{array}$ & - & ONGs/OSCIPs & Gestão & $\begin{array}{l}\text { ASSOCIAÇÃO } \\
\text { MICO-LEÃO } \\
\text { DOURADO ([s/d]) }\end{array}$ \\
\hline 25 & $\begin{array}{l}\text { Consolidação da } \\
\text { Gestão do Uso Público } \\
\text { nas Unidades de } \\
\text { Conservação } \\
\text { Estaduais do Estado } \\
\text { do Rio de Janeiro }\end{array}$ & $\begin{array}{l}12 \text { UCs estaduais do } \\
\text { estado do Rio de } \\
\text { Janeiro }\end{array}$ & - & $\begin{array}{l}\text { ONGs/OSCIPs, Órgãos } \\
\text { governamentais }\end{array}$ & $\begin{array}{l}\text { Capacitação } \\
\text { Gestão } \\
\text { Infraestrutura }\end{array}$ & $\begin{array}{l}\text { PELLEGRINI, 2013; } \\
\text { INSTITUTO TERRA } \\
\text { DE PRESERVAÇÃO } \\
\text { AMBIENTAL ([s/d]) }\end{array}$ \\
\hline 26 & $\begin{array}{l}\text { Projeto Ecossistemas } \\
\text { Costeiros }\end{array}$ & $\begin{array}{l}\text { UCs do litoral do } \\
\text { estado de São } \\
\text { Paulo }\end{array}$ & - & Universidades/Institutos & Capacitação & LOPES (2017) \\
\hline
\end{tabular}

